\title{
NPY Deficiency Prevents Postmenopausal Adiposity by Augmenting Estradiol-Mediated Browning
}

\section{Seongjoon Park, PhD, ${ }^{1, *}$ Erkhembayar Nayantai, MD, ${ }^{2}$ Toshimitsu Komatsu, PhD, ${ }^{1}$ Hiroko Hayashi, MD, PhD, ${ }^{1}$ Ryoichi Mori, PhD, ${ }^{1}$ and Isao Shimokawa, MD, PhD'}

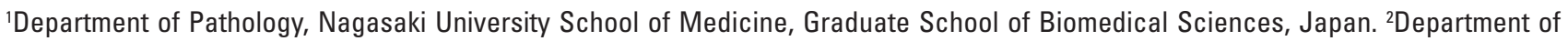
Physiology, School of Biomedicine, Mongolian National University of Medical Sciences, Ulaanbaatar, Mongolia.

*Address correspondence to: Seongjoon Park, PhD, Department of Pathology, Nagasaki University School of Medicine, Graduate School of Biomedical Sciences, 1-12-4 Sakamoto, Nagasaki City 852-8523, Japan. E-mail: psj1026@nagasaki-u.ac.jp

Received: May 23, 2018; Editorial Decision Date: November 27, 2018

Decision Editor: Rozalyn Anderson, PhD

\begin{abstract}
The orexigenic hormone neuropeptide Y (NPY) plays a pivotal role in the peripheral regulation of fat metabolism. However, the mechanisms underlying the effects of sex on NPY function have not been extensively analyzed. In this study, we examined the effects of NPY deficiency on fat metabolism in male and female mice. Body weight was slightly decreased, whereas white adipose tissue (WAT) mass was significantly decreased as the thermogenic program was upregulated in NPY ${ }^{-1}$ female mice compared with that in wild-type mice; these factors were not altered in response to NPY deficiency in male mice. Moreover, lack of NPY resulted in an increase in luteinizing hormone (LH) expression in the pituitary gland, with concomitant activation of the estradiol-mediated thermogenic program in inguinal WAT, and alleviated age-related modification of adiposity in female mice. Taken together, these data revealed a novel intracellular mechanism of NPY in the regulation of fat metabolism and highlighted the sexual dimorphism of NPY as a promising target for drug development to reduce postmenopausal adiposity.
\end{abstract}

Keywords: Adipose tissue, Fat metabolism, Estrogen

Fat is the most concentrated energy source in mammals, providing more than twice as much potential energy as carbohydrates or proteins when energy is insufficient (1). However, abnormally high or low amounts of body fat alter metabolic homeostasis, leading to induction of lipid disorders, such as obesity and lipodystrophy $(2,3)$, particularly in postmenopausal women, who are at increased risk of obesity-related diseases and death (4).

Neuropeptide Y (NPY) is expressed in the hypothalamus with agouti-related peptide (Agrp) and functions to regulate the energy balance in the brain (5). NPY stimulates feeding and weight gain, thereby promoting adiposity when administered or overexpressed in the brain $(6,7)$. Consistent with its role as an orexigenic peptide, NPY is increased during fasting or caloric restriction and suppressed by feeding, leptin, and insulin (5). Conversely, suppression of NPY signaling decreases weight gain and adiposity in obese mice $(8,9)$.

Because estrogen receptors are expressed in NPY neurons, their crosstalk with estrogen and NPY regulates feeding and reproduction (10). Estrogen is not only involved in the development and maintenance of sexual and reproductive function in women but also exerts a wide range of biological effects in both women and men (11,12). Several published papers have demonstrated that estrogen plays protective roles against oxidative stress, muscle damage, adiposity, osteoporosis, and cardiovascular disease (13-17). These studies suggest that loss of estrogen after menopause can significantly increase the risk of many disorders.

We recently found that NPY antagonism reduces adiposity and attenuates age-related imbalances in adipose tissue metabolism in female mice (18). However, the mechanisms underlying the sexual dimorphism of NPY in adipose tissue metabolism are currently unknown. Therefore, in this study, we aimed to determine the sexual dimorphic role of NPY in male and female NPY-- mice. Our results provided important insights into the potential applications of NPY as a new drug target to prevent postmenopausal adiposity.

\section{Methods}

Animals

The animal care and experimental protocols were approved by the Ethics Review Committee for Animal Experimentation at Nagasaki 
University. Male NPY ${ }^{-/}$mice (129S-Npytm1Rpa/J) and female NPY ${ }^{+/+}$ mice (129S6/SvEvTac) were obtained from Jackson Laboratory (Bar Harbor, ME) and Taconic Farms, Inc. (Germantown, NY). They were bred in a barrier facility at the Center for Frontier Life Sciences at Nagasaki University. NPY-null allele maintained on a mixed genetic background derived from intercrosses between the $\mathrm{NPY}^{+/+}$mice (129S6/SvEvTac) and NPY ${ }^{-/}$mice (129S-Npytm1Rpa/J). Three mice were housed in individual cages in the barrier facility (temperature, $21-24^{\circ} \mathrm{C} ; 12$ hours light/dark cycle) under specific pathogen-free conditions, which were maintained for the entire study. All mice were fed ad libitum (AL) with Charles River Formula 1 (CRF-1) diet (Oriental Yeast Co. Ltd., Tokyo, Japan). At 6 months of age, percentage of body fat was measured using 3D micro-CT (Rigaku Co., Tokyo, Japan) after mice were divided into control and letrozole-treated groups. letrozole (Tocris Bioscience, Minneapolis, MN) was provided in drinking water (at a concentration of $0.02,0.1 \mathrm{mg}$ / $\mathrm{kg}$ ) and replaced thrice a week. Body weight was monitored every 2 weeks between 6 and 12 weeks of age and every 4 weeks thereafter. At 10 months old, percentage of body fat was measured using 3D micro-CT (Rigaku Co.).

After serum samples collected from cardiac puncture, 2-, 6-, and 22 -month old female mice were sacrificed, and tissues were immediately collected.

\section{Estradiol Quantification}

Serum estradiol concentration was determined using ELISA system (Cayman chemical, Michigan).

\section{Western Blotting}

Antibodies for UCP1 were obtained from Abcam (Cambridge, UK), and Anti- $\beta$-actin was purchased from Cell Signaling Technology (Danvers, MA). Enhanced chemiluminescence (ECL) western blotting detection reagents and ECL-anti-rabbit or mouse IgG, horseradish peroxidase-linked species-specific whole antibodies were purchased from Amersham Pharmacia Biotech (Little Chalfont, UK).

All samples were boiled for 3 minutes and chilled on ice. Proteins were separated by SDS-PAGE and transferred to a nitrocellulose membrane. Membrane were immediately placed in blocking solution $(2 \%$ bovine serum albumin [BSA] or $5 \%$ nonfat dry milk in trisbuffered saline with $0.1 \%$ tween 20 [TBS-T] buffer) for 1 hour and incubated with the primary antibody for overnight at $4{ }^{\circ} \mathrm{C}$ followed by the second antibody for 1 hour at room temperature. Antibody labeling was detected using ECL as per the manufacturer's instructions. Specific signals were quantified by Fluorchem (DE500-5T, Alphainnotech Corporation, San Leandro, CA) with associated image analysis software, AlphaEase FC.

\section{Quantitative Real-Time PCR}

Total RNA was isolated using RNeasy tissue kit (Qiagen, CA) and cDNA was synthesized using ReverTra qPCR RT kit (Toyobo, Osaka, Japan). The relative amount of mRNA expression was analyzed by quantitative polymerase chain reaction (qPCR) with THUNDERBIRD SYBR qPCR Mix (Toyobo, Osaka, Japan) according the protocol provided by the manufacturer. The results were normalized by the $18 \mathrm{~S}$ expression levels. See Supplementary Table 1 for primer sequences.

\section{Histology}

Adipose tissue was fixed in $4 \%$ paraformaldehyde and embedded in paraffin. The tissue was then cut into $5-\mu \mathrm{m}$-thick sections and incubated with anti-rabbit UCP1 (Abcam) antibody at $4^{\circ} \mathrm{C}$ overnight. For fluorescent detection, sections were incubated with Alexa fluor 488-labeled anti-rabbit antibody (Invitrogen) for 2 hours and counterstained with 4', 6-diamidino-2-phenylindole (DAPI). Adipocyte size was determined with paraffin sections of adipose tissue stained with hematoxylin and eosin. After staining, the mean diameter was calculated in adipose tissue from mice using NISElements AR software (Nikon Corp). Samples were visualized using a confocal microscope (C2+ system; Nikon Corp., Tokyo, Japan). Digital images were acquired by NIS-Elements AR software.

\section{Body Temperature}

The body temperature of mice was measured with rectal thermometers (TERUMO CTM-303: Terumo Corporation, Tokyo). The rectal probe (ME-PDK061: Terumo Corporation) was lubricated with glycerol and then inserted into the rectum about $3 \mathrm{~cm}$. The rectal temperature measurement procedure took 1 minute.

\section{Cell Culture and Induction of Adipocyte Differentiation}

3T3-L1 cells were cultured in Dulbecco's modified Eagle's medium (DMEM) containing high glucose $(25 \mathrm{mM})$ and supplemented with $10 \%$ calf serum. Differentiation was induced 2 days after confluence (Day 0) by adding an induction cocktail containing $100 \mathrm{nM}$ insulin, $1 \mu \mathrm{M}$ dexamethasone, and $0.5 \mathrm{mM} 1$-methyl-3-isobutyl-xanthine in DMEM/F12 medium containing 10\% fetal bovine serum (FBS) for 3 days. On Day 3, differentiation medium was replaced with $10 \%$ FBS DMEM/F12 medium containing with $100 \mathrm{nM}$ insulin for additional 4 days, and then media were changed every 2 days. Estradiol and genistein were treated when media were changed. All media contained $100 \mathrm{U} / \mathrm{mL}$ penicillin, and $100 \mu \mathrm{g} / \mathrm{mL}$ streptomycin. All cells were maintained and differentiated at $37^{\circ} \mathrm{C}$ in a humidified $5 \% \mathrm{CO}_{2}$.

\section{Cell Proliferation Assay}

Proliferation capacity of 3T3-L1 preadipocytes and adipocytes was measured with WST1 colorimetric assay kit (Roche, Mannheim, Germany).

\section{Oil Red O Staining}

After differentiation, cells were washed with phosphate-buffered saline, fixed with $4 \%$ paraformaldehyde, in phosphate-buffered saline for 1 hour. Cells were washed with $60 \%$ isopropanol and stained with Oil red $\mathrm{O}$ working solution $(6: 4,0.2 \%$ Oil red $\mathrm{O}$ dye in $\mathrm{H}_{2} \mathrm{O}$ ) for 15 minutes. Cells were washed four times with $\mathrm{H}_{2} \mathrm{O}$ and images were photographed. Finally, Oil red O staining was dissolved in $200 \mu \mathrm{L}$ of isopropanol, and the absorbance measured at $520 \mathrm{~nm}$ using a microplate reader.

\section{Statistical Analysis}

All data are presented as means \pm SEM and were analyzed by unpaired two-tailed Student's $t$ test and two-way analysis of variance (ANOVA) test. Statistical analysis was performed with GraphPad Prism 5 and $p<.05$ was considered significant.

\section{Results}

\section{NPY Deficiency Reduced Adiposity in Female Mice But Not in Male Mice}

In order to investigate whether the phenotypes of mice were modified by NPY deficiency, we analyzed body weight (BW), fat mass, 
and food intake (FI) in 6-month-old female and male mice. No differences in BW and FI were observed between $\mathrm{NPY}^{-}$and wildtype (wt) mice. Two-way (genotype and gender) ANOVA analyses performed in each strain showed a significant effect of gender (BW and FI, $p<.001$ ), but not genotype or gender and genotype interaction (Figure 1A). Interestingly, the amounts of visceral (gonadal,
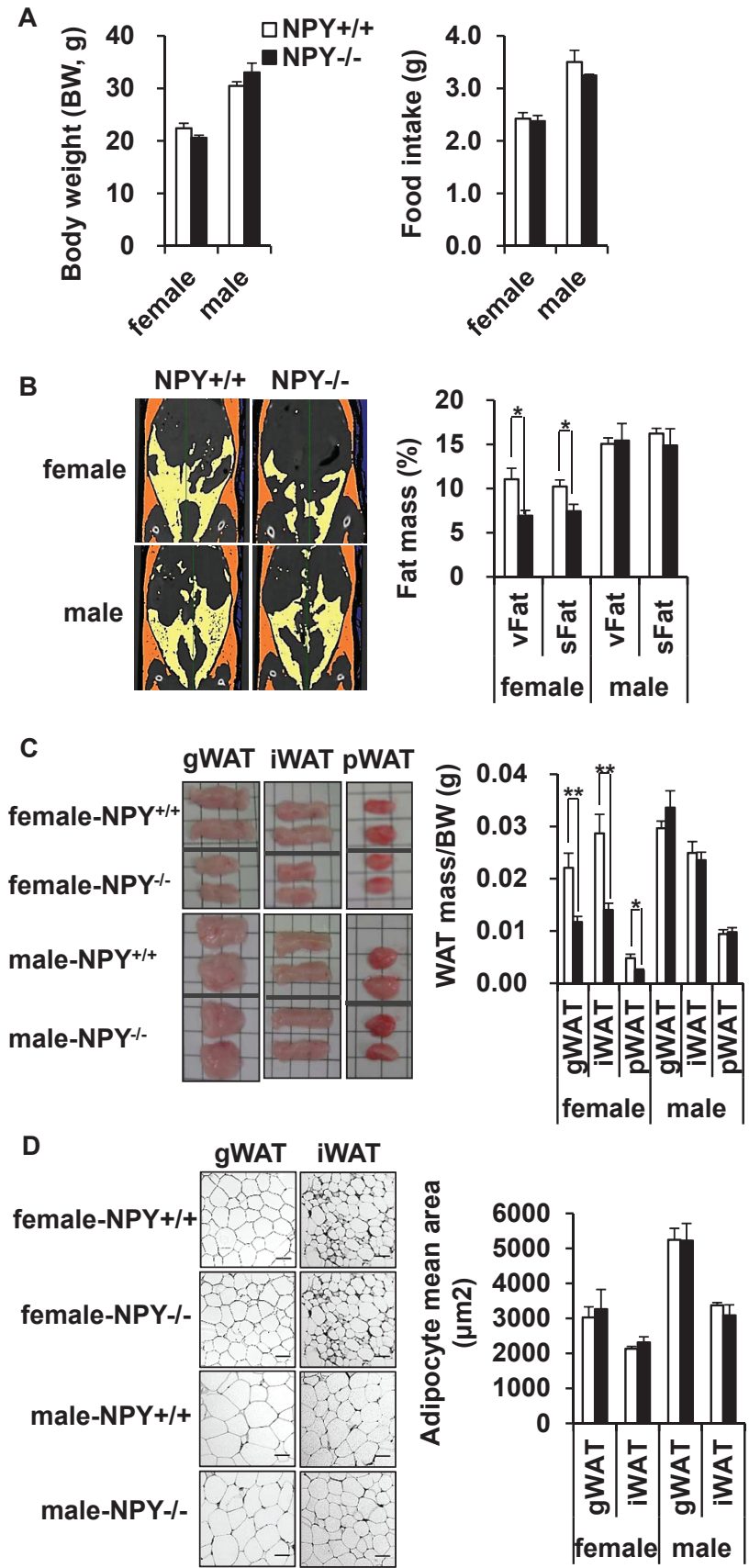

Figure 1. NPY deficiency reduces adiposity in female mice, but does not affect in male mice. (A) Body weight and food intake measurements in $\mathrm{NPY}^{+/+}$and $\mathrm{NPY}^{-/-}$mice at 6 months of age. (B) 3D-Micro CT analysis for the percentage of visceral ( $v$ ) and subcutaneous (s) fat in same mice. (C) White adipose tissue (WAT) depot weights measurements in same mice. (D) Representative images from immunohistochemistry for H\&E stain in gWAT and iWAT (left panel), and the mean sizes of adipocytes (right panel). All data are presented as the mean \pm SEM, $n=4-6$ per group; ${ }^{*} p<.05$ and ${ }^{* *} p<.01$. Scale bars: $50 \mu \mathrm{m}$. perirenal) and subcutaneous (inguinal) adipose tissues in NPY $\%$ mice differed between male and female mice. Micro-CT analysis of body composition demonstrated that the visceral $(\mathrm{v})$ and subcutaneous $(\mathrm{s})$ adipose tissue mass differed according to genotype, with a significantly reduction observed in female NPY ${ }^{-/}$mice compared with that in female wt mice; there was no difference between genotypes in male mice (Figure 1B). Two-way (genotype and gender) ANOVA analyses performed in each strain showed a significant effect of gender (vAT and sAT, $p<.001$ ), but not genotype or gender and genotype interaction (Figure 1B). To further examine the fat reduction in NPY $\mathrm{fe}^{-}$ male mice, we measured the amounts of gonadal $(\mathrm{g})$, perirenal $(\mathrm{p})$, and inguinal (i) adipose tissues after mice were sacrificed. In parallel to results of micro-CT, the amount of adipose tissue in NPY ${ }^{-}$mice was marked decreased in female mice, whereas no difference between genotypes was observed in male mice (Figure 1C). Two-way (genotype and gender) ANOVA analyses performed in each strain showed a significant effect of gender (gWAT and pWAT, $p<.001$ ), but not genotype or gender and genotype interaction (Figure 1C). The adipocyte sizes in gWAT and iWAT were not modified by NPY deficiency, although both were higher in male mice than in female mice (gWAT, $p$ $<.01$; iWAT, iWAT, $p<.001$; Figure 1D). These data showed that the regulation of adiposity by NPY antagonism was dependent on sex.

\section{NPY Deficiency Increased Thermogenesis in Female Mice But Not in Male Mice}

Reduction of WAT mass (Figure 1B and C) without changes in food intake (Figure 1A) suggested an increase in energy utilization in NPY1- female mice. In previous studies, we showed that mRNA expression of genes involved in thermogenesis and oxidative phosphorylation was significantly upregulated in iWAT from NPY ${ }^{--}$female mice (18). Furthermore, the expression of these genes was more dynamically regulated in iWAT than interscapular brown adipose tissue (BAT) by NPY deficiency (18). Therefore, we next analyzed the expression of uncoupling protein 1 (UCP1), which is a key regulator involved in thermogenesis in the iWAT of both sexes. The results from immunostaining and western blotting showed that the expression level of UCP1 was elevated in NPY $\%$ female mice, but no difference between genotypes was observed in male mice (Figure 2A and B). In addition, female mice had a significantly higher UCP1 level than male mice (Figure 2A). In female mice, H\&E-stained sections of BAT showed slightly reduced fat droplets within the vast majority of the brown adipocytes; however, no noticeable differences between genotypes were observed in male mice (Figure 2C). The amount of brown adipose tissue showed no difference between genotypes in female mice, but was slightly increased in $\mathrm{NPY}^{-/}$mice compared with that in $\mathrm{NPY}^{+/+}$male mice (Supplementary Figure S1). The protein expression level of UCP1 in BAT was not different between genotypes in female and male mice (Figure 2D). We next explored whether this alteration in thermogenic gene expression is controlled body temperature by evaluating body temperature in the light and dark phases for both sexes. In accordance with increased thermogenic gene expression, body temperature was higher in female $\mathrm{NPY}^{-/}$mice than in female wt mice, particularly during the dark phase (Figure 2E). Furthermore, body temperature was higher in female mice than in male mice during the dark phase (gender, $p<.01$, genotype and gender, $p<.01$ ). However, no differences in the thermogenic program between genotypes were observed in male mice (Figure 2A-E).

\section{NPY Deficiency Increased Estradiol and Reduced Adiposity}

Because estradiol has been shown to enhance energy utilization through upregulation of thermogenesis (19), we hypothesized that 
NPY deficiency may reduce adiposity through activation of estradiol-mediated thermogenesis in female mice. We observed higher

A

$<$ iWAT >

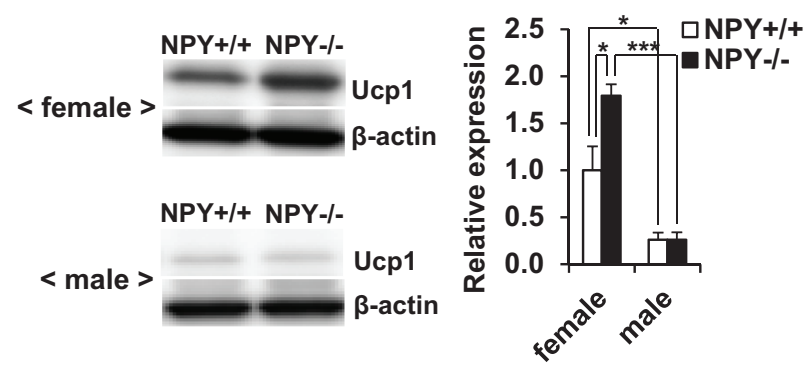

B

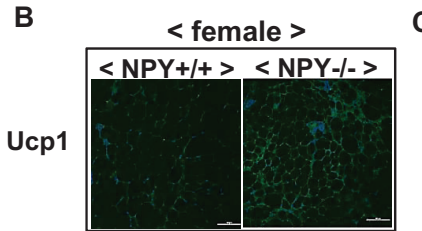

$<$ male $>$

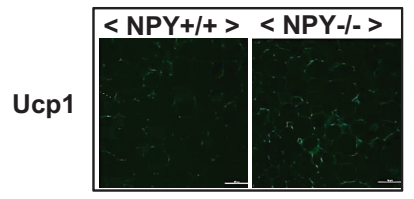

C

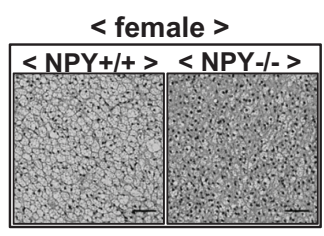

$<$ male $>$

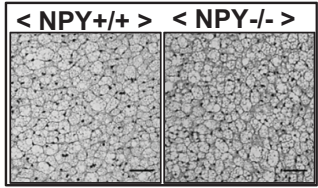

D

<BAT>
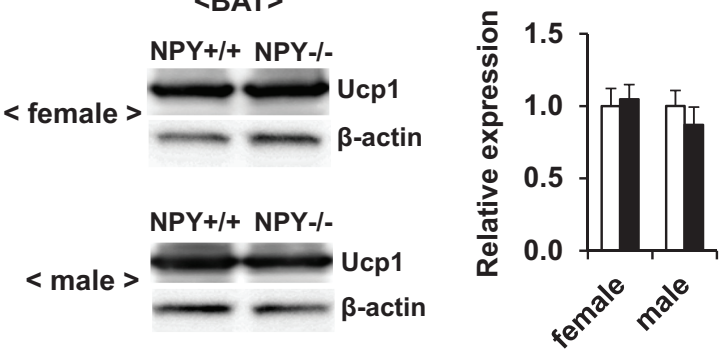

E
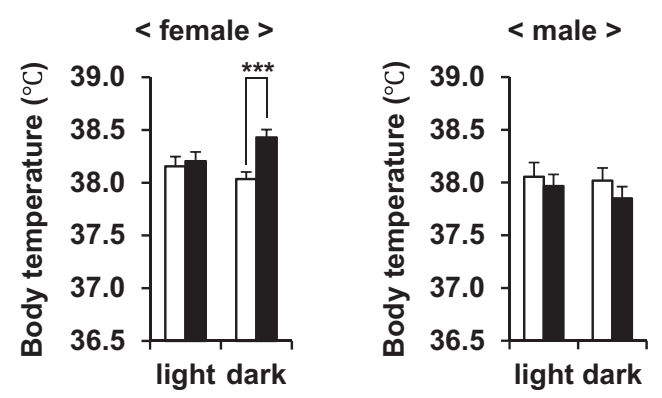

Figure 2. NPY deficiency leads to increase in thermogenesis in female mice, but does not affect in male mice. (A) Western blotting for Ucp1 in iWAT from $\mathrm{NPY}^{+/+}$and $\mathrm{NPY}^{-/-}$mice at 6 months of age (left panel) and quantified (right panel), $n=6$ per group. (B) Microscopic images of iWAT stained with antibody recognizing Ucp1 as indicated with nuclei stained with 4', 6-diamidino2-phenylindole (DAPI), $n=6$ per group. (C) Representative images from immunohistochemistry for H\&E stain in BAT. (D) Western blotting for Ucp 1 in BAT from NPY+/+ and NPY-/ mice (left panel) and quantified (right panel), $n=6$ per group. (E) Rectal body temperature in same mice, $n=10-11$ per group. All data are presented as the mean \pm SEM; ${ }^{*} p<.05$ and ${ }^{* * *} p<.001$. Scale bars: $100 \mu \mathrm{m}$. BAT $=$ Brown adipose tissue; $\mathrm{WAT}=$ White adipose tissue. serum estradiol levels in female $\mathrm{NPY}^{\star}$ mice than in female wt mice (Figure 3A), whereas no differences between genotypes were observed in male mice (Supplementary Figure S2). To determine whether the increase in estradiol caused the reduced adiposity in female $\mathrm{NPY}^{-/}$mice, mice were treated with letrozole, an aromatase

\section{A}
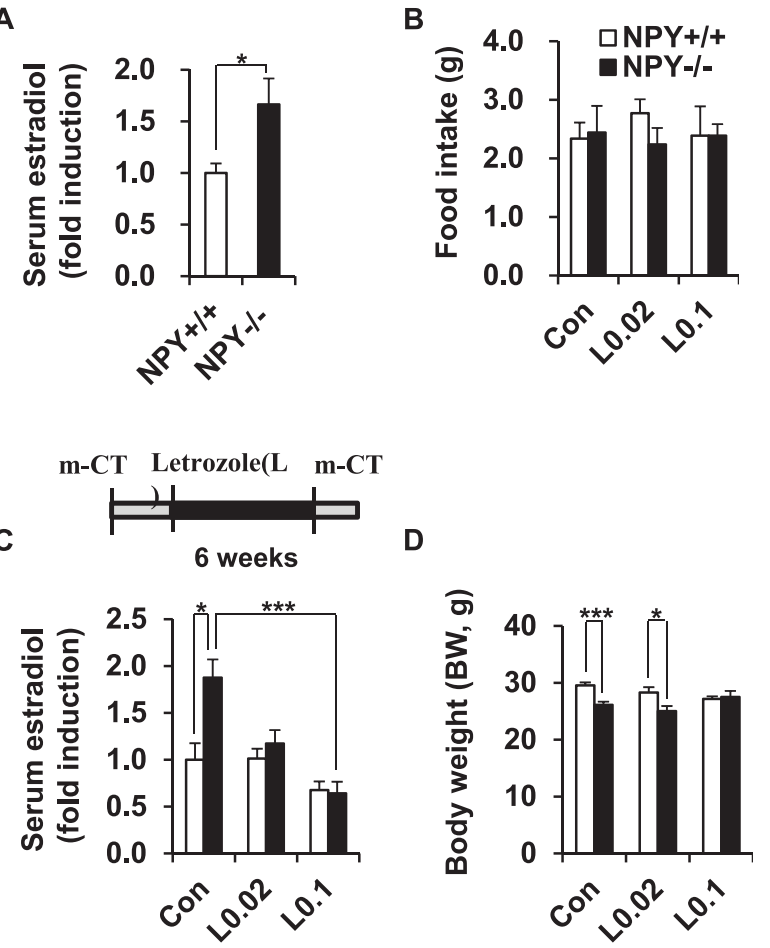

E
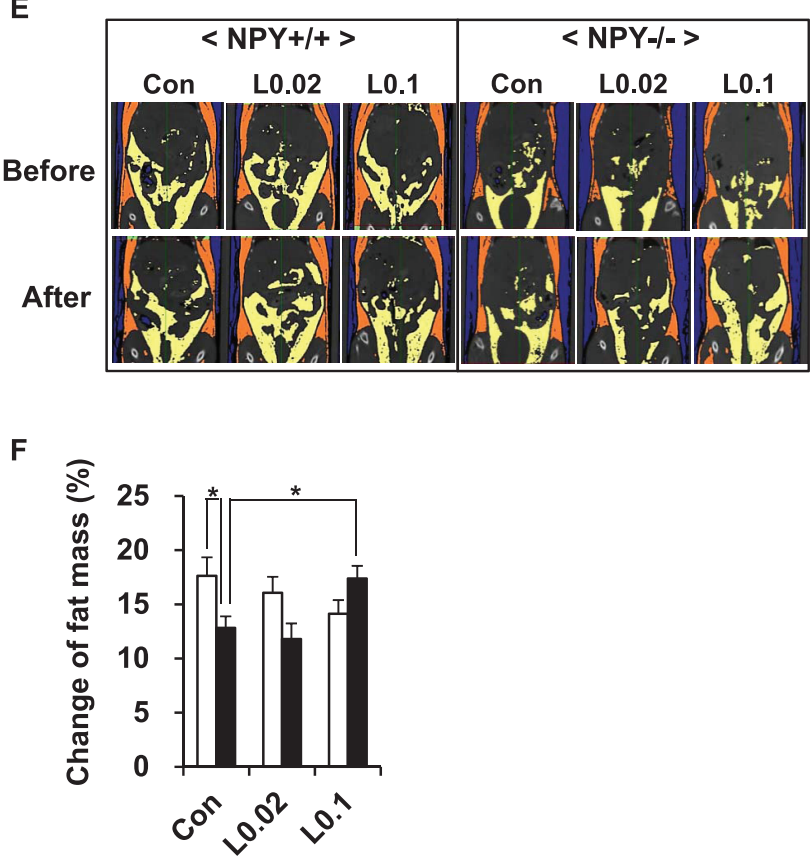

Figure 3. NPY deficiency increases estradiol and reduces adiposity, which is inhibited by letrozole. $(\mathbf{A}, \mathbf{C})$ Serum estradiol level in $\mathrm{NPY}^{+/+}$and $\mathrm{NPY}^{-/}$mice at 6 months of age and letrozole-treated mice. (B,D) Food intake and body weight measurements in letrozole-treated mice. (E,F) 3D-Micro CT analysis for the percent change of fat mass in letrozole-treated mice. All data are presented as the mean \pm SEM, $n=6-8$ per group; ${ }^{*} p<.05$ and ${ }^{* * *} p<.001$. 
inhibitor, for 6 weeks. Food intake was not altered by letrozole treatment (Figure 3B). Treatment with a high dose of letrozole $(0.1 \mathrm{mg} /$ $\mathrm{kg}$ ) inhibited the increase in serum estradiol and reductions in body weight and fat mass mediated by NPY deficiency. However, letrozole treatment did not affect serum estradiol level, body weight, and fat mass in control mice (Figure 3C-F and Supplementary Figure S3A,B).

\section{Letrozole Inhibited NPY Deficiency-Mediated Upregulation of the Thermogenic Gene Program}

We showed that NPY deficiency enhanced thermogenesis in female mice (Figure 2). In a previous report, we observed that the change in expression of genes involved in thermogenesis and oxidative metabolism was much higher in iWAT than in BAT (18). To investigate whether this activation of thermogenic program mediated by NPY deficiency resulted from upregulation of estrogen, we analyzed thermogenic program after letrozole treatment. The adipocyte sizes in iWAT were not modified by NPY deficiency in the control group, but were increased by NPY deficiency in letrozole treatment group (Figure 4A). The adipocyte sizes in gWAT were not significantly modified by genotypes or letrozole treatment (Supplementary Figure S4). We also found that mRNA levels of Ucp1, cytochrome C oxidase 7a1 (COX7a1), COX8b, peroxisome proliferator-activated receptor alpha $(P P A R \alpha)$, and deiodinase 2 (Dio2), which are involved in thermogenesis and oxidative metabolism, were higher in iWAT from NPY ${ }^{-/}$mice than in that from wt mice (Figure 4B). Moreover, these alterations were inhibited by letrozole treatment. Consistent with the mRNA results, the level of UCP1 protein in iWAT was increased in $\mathrm{NPY}^{-}$mice, and this change was reversed by letrozole treatment (Figure 4C). However, letrozole treatment did not significantly affect the fat accumulation and Ucp1 expression in BAT (Figure 4D and E). Estrogens are regulated by gonadotropin-releasing hormone $(\mathrm{GnRH})$, luteinizing hormone $(\mathrm{LH})$, and follicle-stimulating hormone (FSH) (20), and NPY inhibits neuronal GnRH activity (21). Based on these findings, we determined the mRNA levels of LH and FSH to verify the mechanisms underlying the effects of NPY on the regulation of estradiol. The mRNA levels of FSH and LH in the pituitary gland demonstrated that lack of NPY increased estradiol level through modification of pituitary gonadotropin expression, although the expression of FSH was marginally increased (Figure 4F). Taken together, these data suggested that reduction of adiposity in female NPY ${ }^{-/}$mice was due to the activation of LH-estradiol-mediated thermogenesis in iWAT.

\section{Estradiol Altered the Expression of Genes Related to Adipocyte Differentiation and Beige Adipocytes}

To further explore the functional roles of estradiol in adipocyte metabolism, we used 3T3-L1 preadipocytes in subsequent experiments. 3T3-L1 preadipocytes showed increased mRNA levels of adipogenic/lipogenic genes, such as PPAR $\gamma 2$, Fatty acid binding protein 4 (ap2), and Fatty acid synthase (FAS), as well as higher accumulation of lipid droplets after differentiation (Supplementary Figure S4A,C). To establish whether estradiol regulated adipogenesis/lipogenesis and thermogenesis, we analyzed the mRNA expression of adipogenic/lipogenic/thermogenic genes after treatment with estradiol. Because genistein has been shown to regulate adipocyte metabolism (22), we also added genistein as a positive control. Indeed, 50 and $100 \mu \mathrm{M}$ estradiol inhibited lipid accumulation and mRNA expression of adipogenic/lipogenic genes, such as PPAR 2 , ap2, and FAS, whereas genistein only affected the expression of these genes when used at a high dose (Supplementary Figure S5A,C). Adipogenic/lipogenic action can be augmented by increases in cell proliferation.
A

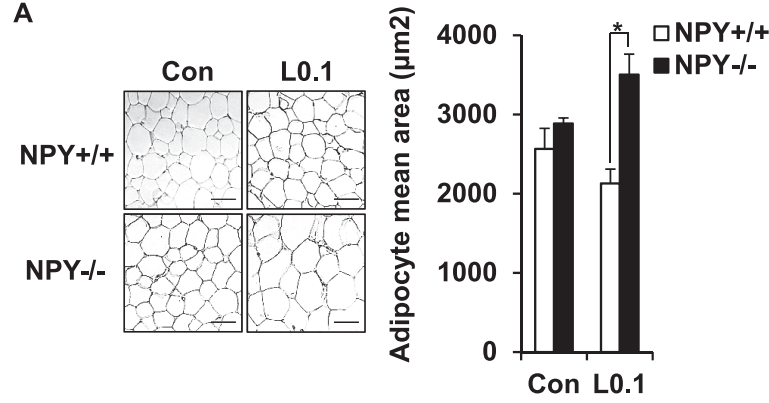

B

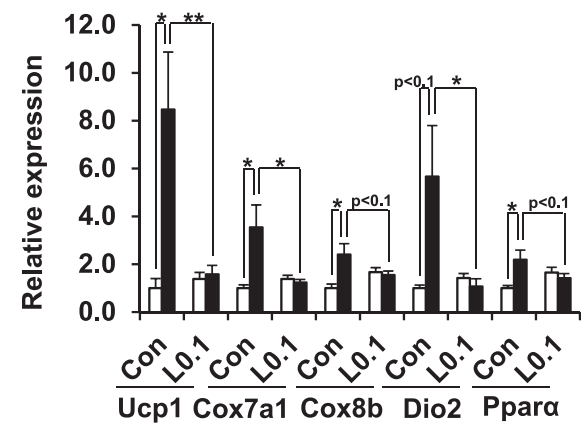

C

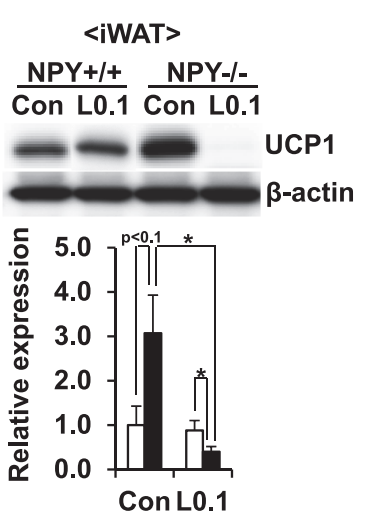

D

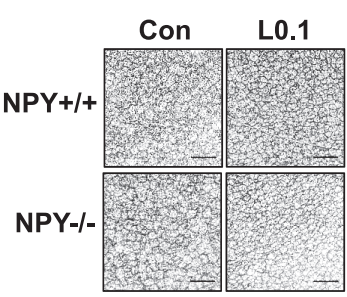

E
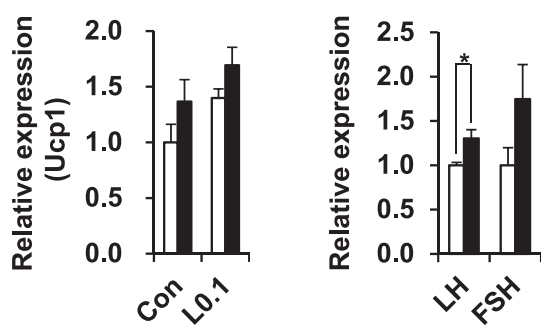

Figure 4. Letrozole inhibits NPY deficiency-mediated upregulation of thermogenic gene program. (A) Representative images from immunohistochemistry for H\&E stain in iWAT (left panel), and the mean sizes of adipocytes (right panel) in letrozole-treated mice. Scale bars: $50 \mu \mathrm{m}$. (B) mRNA expression levels of Ucp1, Cox7a1, Cox8b, Dio2, and Ppara in iWAT from same mice. (C) Western blotting for UCP1 in iWAT and BAT from same mice (left panel) and quantified (right panel). (D) Representative images from immunohistochemistry for H\&E stain in BAT from same mice. Scale bars: $100 \mu \mathrm{m}$. (E) mRNA expression levels of Ucp1 in BAT from same mice. (F) mRNA expression levels of $F S H$ and $L H$ in pituitary gland from same mice. All data are presented as the mean \pm SEM; $n=6-8$ per group; ${ }^{*} p<.05$ and ${ }^{* *} p<.01$. BAT $=$ Brown adipose tissue; WAT $=$ White adipose tissue.

To study whether the estradiol-mediated inhibition of adipogenic/lipogenic action resulted from reduced cell proliferation, we 
performed cell proliferation assays. Both low and high concentrations of estradiol did not affect cell proliferation, while high dose of genistein significantly inhibited cell proliferation (Supplementary Figure S5B).

3T3-L1 preadipocytes can be altered to beige/brown adipocytes by genistein, $\beta$ adrenergic activation, and other stimulations $(22,23)$. Therefore, we next examined the possibility that estradiol may transform 3T3-L1 preadipocytes into beige/brown adipocytes. Indeed, 50 and $100 \mu \mathrm{M}$ estradiol induced beige and/or brown adipocyte markers, such as peroxisome proliferator-activated receptor gamma coactivator-1alpha $(P g c 1 \alpha)$, TNF-receptor superfamily 9 (Cd137), Short stature homeobox 2 (Shox2), and Dio2, similar to genistein (Supplementary Figure S5D). Interestingly, the brown adipocyte-specific marker, LIM homeobox protein 8 (Lhx8) was significantly increased by estradiol, but not by genistein (Supplementary Figure S5D). These data indicated that estradiol was a stronger regulator of browning in adipocytes than genistein.

\section{NPY-/- Female Mice Displayed Increased Estradiol Levels and Decreased Adiposity During Aging}

Body fat is increased after menopause, which leads to increased risk of metabolic syndrome $(24,25)$. To assess whether NPY deficiency rescued adiposity in middle-aged female mice, we monitored agerelated changes in body weight, food intake, and fat mass. Food intake was not modified by genotype (Supplementary Figure S6). Body weight was marginally reduced, and white adipose tissue (WAT) mass was significantly decreased in female NPY ${ }^{\star-}$ mice compared with that in female $\mathrm{NPY}^{+/+}$mice during aging (Figure $5 \mathrm{~A}$ and $\mathrm{B})$; however, no changes were observed in male mice (Supplementary Figures S6B and S7A). Furthermore, serum estradiol levels were decreased in 22-month-old mice, whereas NPY deficiency rescued the reduction in estradiol (Figure 5C); there were no genotype- or age-related differences in male mice (Supplementary Figure S7B).

The crosstalk between estrogen and NPY not only regulates feeding and reproduction but also controls adipocyte metabolism $(10,26)$. Notably, estrogen is negatively correlated with NPY $(26,27)$. To determine whether increased serum estradiol was associated with peripheral expression of NPY, we measured the mRNA expression of Npy in iWAT and gWAT from young and middle-aged female mice. We found that NPY expression in iWAT was inversely correlated with serum estradiol levels, but it was not in gWAT (Figure 5C and D and Supplementary Figure S8). The balance between estrogen receptor (ER) $\alpha$ and $E R \beta$ in adipose tissue is thought to be important for the development of metabolic diseases (24). Therefore, we analyzed mRNA levels of $E R \alpha$ and $E R \beta$ in iWAT of female mice. ER $\alpha$ was not altered by aging or genotype, whereas $\operatorname{ER} \beta$ was significantly increased in 22-month-old mice; this increase was revered by NPY deficiency (Figure 5E).

\section{Discussion}

Body fat increases with age and the levels of triglycerides, as the major component of fat, are high in middle-aged animals, leading to increased risk of metabolic diseases and suggesting that fat in middle age is critical target for the prevention of obesity. Numerous in vivo studies have shown the importance of estradiol in glucose homeostasis and obesity $(15,28)$. In addition, clinical studies have shown a correlation between estradiol and several aspects of metabolic syndrome (29). However, the detailed mechanisms of communication between central and peripheral tissue, particularly in adipose tissue, are unknown. Moreover, although NPY antagonism plays a key role in the regulation of adiposity $(9,18)$, the specific molecular
A

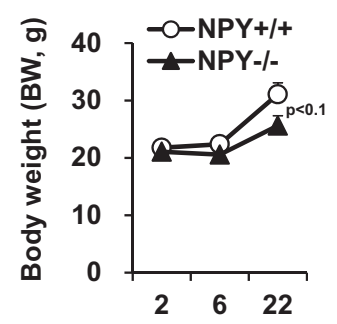

C

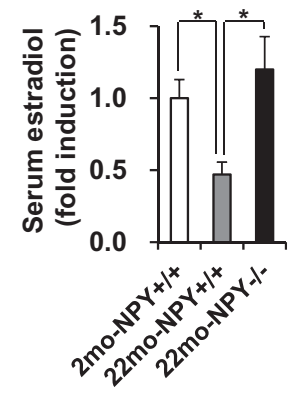

B

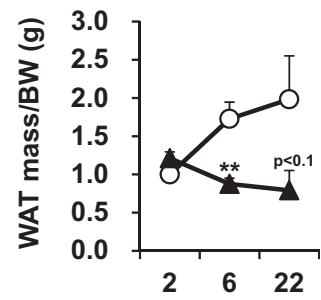

D

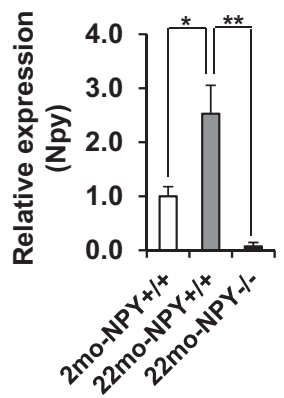

E

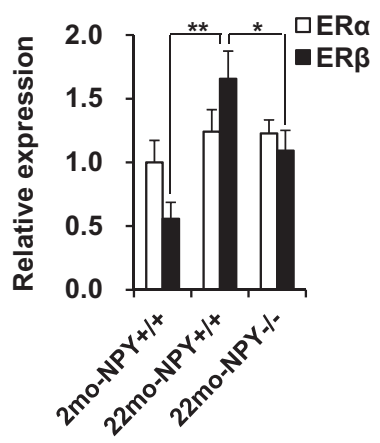

$\mathbf{F}$

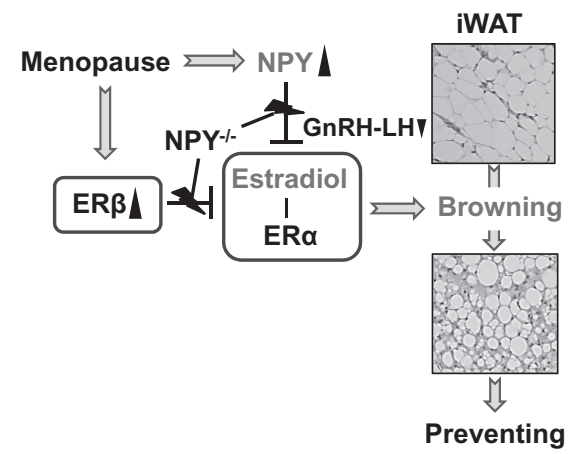

Figure 5. $\mathrm{NPY}^{-1}$ female mice display increase in estradiol level as well as decrease in adiposity during aging. (A) Body weight and (B) white adipose tissue (WAT) depot weights measurements in $\mathrm{NPY}^{+/+}$and $\mathrm{NPY}^{-/-}$mice at 2,6, and 22 months of age, $n=5-6$ per group. (C) Serum estradiol level and (D, E) mRNA expression levels of $N p y, E R \alpha$, and $E R \beta$ in iWAT from NPY+/+ and NPY1- mice at 2 and 22 months of age, $n=3-6$ per group. All data are presented as the mean $\pm \mathrm{SEM} ;{ }^{*} p<.05$ and ${ }^{* *} p<.01$. (F) Model summarizing the interaction of NPY and estrogen in postmenopausal obesity. NPY deficiency ameliorates age-related decrease of white fat browning through modification of luteinizing hormone-estrogen axis which may contribute to preventing postmenopausal obesity.

mechanisms through which NPY is linked to adiposity in the postmenopausal period are unknown. The results of the present study demonstrated that genetic deletion of NPY reduced adiposity by 
the activation of estrogen-mediated thermogenesis in middle-aged female mice.

Female NPY ${ }^{-/}$mice exhibited a significant reduction in adiposity and increases in adipose (iWAT)-related thermogenic gene expression and body temperature. Because the expression of Ucp1 was not significantly modified by NPY deficiency in BAT (Figure 2D), we focused on iWAT in this study. In contrast with female mice, both thermogenesis and adiposity were unaffected by NPY deletion in male mice, in contrast to other studies showing increased thermogenesis and reduced adiposity in NPY-knockout rats (8). This difference could be due to the use of global NPY ${ }^{-}$in our experiments versus a dorsomedial hypothalamic NPY ${ }^{-/}$model in rats. Moreover, the effects of NPY deficiency on adiposity were much higher in rats fed a high-fat diet than in those fed a control diet in their study. Moreover, in a previous report, NPY and its receptors were found to be expressed not only in the hypothalamus but also in adipose tissues (18); therefore, both hypothalamic and peripheral regulation of NPY signaling may affect this discrepancy. Our results were consistent with the findings of Erichson et al., who demonstrated that the body fat was not modified by global NPY ${ }^{-1}$ in male mice (30).

We showed that the expression of the thermogenic protein, Ucp1, was upregulated in iWAT of female NPY ${ }^{\star-}$ mice compared with that in wt mice, but it was not modified in male mice. To examine whether the deletion of NPY promoted white fat browning through upregulation of estradiol, we evaluated mRNA levels of brown/beige adipocyte-specific markers in 3T3-L1 adipocytes after treatment with estradiol. Our results showed that 50 and $100 \mu \mathrm{M}$ estradiol increased mRNA levels of brown and/or beige adipocyte-specific genes, such as Pgc1a, Cd137, Shox2, Dio2, and Lhx 8 (Supplementary Figure S4D). This indicated that upregulation of estradiol was a cause of the enhanced thermogenesis observed in $\mathrm{NPY}^{\star-}$ mice. Our results were consistent with the finding that 3T3-L1 preadipocytes could be transformed to beige/brown adipocytes by genistein or other stimulants $(22,23)$, although the effects of estradiol were not evaluated in previous studies. Notably, both estradiol and genistein did not affect the expression of the well-known brown/beige adipocyte marker Ucp1 in 3T3-L1 preadipocytes (data not shown). Aziz et al. showed that the mRNA level of Ucp1 was increased on Day 12 after induction of differentiation in the presence of genistein; therefore, this result may be related to the use of shortterm treatment with estradiol or genistein ( 8 days).

Estrogen receptors are expressed in NPY neurons and their crosstalk with estrogen and NPY not only regulates feeding and reproduction but also controls adipocyte metabolism $(10,26)$. Interestingly, recent studies have shown that estrogen is negatively correlated with NPY $(26,27)$. In support of these results, we found that serum estradiol levels were upregulated in female $\mathrm{NPY}^{-1-}$ mice compared with that in wt mice (Figure 3A and $\mathrm{C}$ and Figure 5C). Moreover, the aromatase inhibitor, letrozole, inhibited the metabolic phenotype of female $\mathrm{NPY}^{-/}$mice, such as reduced adiposity and increased thermogenesis. Because we analyzed the metabolic phenotype of mice at 13 months, the age at which transition to reproductive senescence occurs (31), letrozole did not affect the metabolic phenotype of the control mice (Figures 3 and 4). These results clearly showed that NPY deficiency reduced adiposity by increasing thermogenesis via upregulation of estradiol in female mice. However, serum estradiol levels and adiposity in middle-aged male mice were not modified by NPY deficiency (Supplementary Figures S1 and S6A). Accordingly, we propose that global knockout of NPY may not affect adiposity in male mice and that NPY could be involved in reducing serum estradiol levels.
Estradiol has been reported to activate thermogenesis in BAT (19). However, increase in estradiol due to NPY deficiency did not affect the major thermogenic protein, Ucp1, in the current study (Figure 2D). Under basal conditions, circulating estradiol concentration is approximately $5-140 \rho \mathrm{g} / \mathrm{mL}$ in female rodents (32), whereas serum estradiol level in NPY ${ }^{-/}$mice was approximately $9 \rho \mathrm{g} / \mathrm{mL}$. However, the thermogenic activation in BAT reported previously was induced by s.c. injection of extremely high dose of estradiol $(0.2 \mu \mathrm{g} / \mathrm{mL})$ for 7 days. Therefore, further studies were performed to confirm whether treatment with physiological level of estradiol regulates thermogenesis in BAT. Estrogen has been studied extensively owing to its role as a therapeutic target for the prevention of various metabolic diseases during menopause $(15,28,29)$. However, estrogen replacement therapy has been shown to have diverse effects depending on the applied concentration (33). Thus, recovery of decreased serum estradiol levels in middle-aged mice by NPY deficiency could supply an appropriate functional concentration of estradiol. Furthermore, all women treated with hormone replacement therapy (HRT) do not attain therapeutic levels of estradiol (34). These effects may be related to the expression of ERs, which are important regulators of metabolic diseases (24). ER $\alpha^{-/-}$mice display increased adiposity and insulin resistance and impaired glucose tolerance (35), indicating that $\mathrm{ER} \alpha$ has a protective role in metabolic disorder. Moreover, age-related increases in ER $\beta$ paired with decreased PPAR $\gamma$ may predispose E2-deficiency postmenopausal women to increased adiposity and associated metabolic diseases $(36,37)$, suggesting that ER $\beta$ may have a negative role in metabolic diseases. We found that age-related increases in adiposity and adipose ER $\beta$, concurrent with decreased serum estradiol and adipose thermogenesis (Figure 5), were recovered by NPY deficiency, potentially providing better therapeutic effects than estrogen replacement for older menopausal women. NPY is expressed in both gonadal and inguinal adipose tissue, but its expression in inguinal adipose tissue only increased concurrent with decreases in serum estradiol in middle-aged female mice (Figure 5 and Supplementary Figure S7), suggesting that upregulation of NPY expression in inguinal adipose tissue may be a diagnostic marker of premenopausal obesity.

Collectively, our data provided the first evidence that lack of NPY increases iWAT thermogenesis and reduces adiposity through modification of the LH-estradiol axis. Furthermore, NPY deficiency recovered age-related modifications in adiposity, serum estradiol, and adipose ER $\beta$ expression levels in female mice. The findings of this study also highlighted possible treatment strategies for prevention of postmenopausal obesity (Figure 5F).

\section{Supplementary Material}

Supplementary data is available at The Journals of Gerontology, Series A: Biological Sciences and Medical Sciences online.

\section{Funding}

This work was supported by Grants-in-Aid for Scientific Research from the Japan Society for the Promotion of Science (no. 15H04682 and 15K19517).

\section{Acknowledgments}

We are grateful to the staff at the Biomedical Research Center at the Center for Frontier Life Sciences, Nagasaki University for their technical assistance and animal care. We also thank Chika Matsumoto, Yutaka Araki, and Yuko Moriyama for excellent technical assistance. 


\section{Conflict of Interest}

None reported.

\section{Author Contributions}

S.P. conducted all experiments and wrote the manuscript. T.K. and E.N. helped analysis of 3D-microCT and animal care. H.H., and R.M. served as advisors for histological analysis and animal care. I.S. helped experimental design, data analysis, and interpretation.

\section{References}

1. Lafontan M, Langin D. Lipolysis and lipid mobilization in human adipose tissue. Prog Lipid Res. 2009;48:275-297. doi:10.1016/j. plipres.2009.05.001

2. Lewington S, MacMahon S, Aromaa A, et al. Body-mass index and cause-specific mortality in 900000 adults: collaborative analyses of 57 prospective studies. Lancet. 2009;373:1083-1096. doi:10.1016/ S0140-6736(09)60318-4

3. Mao J, Yang T, Gu Z, et al. aP2-Cre-mediated inactivation of acetyl-CoA carboxylase 1 causes growth retardation and reduced lipid accumulation in adipose tissues. Proc Natl Acad Sci USA. 2009;106:17576-17581. doi:10.1073/pnas.0909055106

4. Dennis KE. Postmenopausal women and the health consequences of obesity. J Obstet Gynecol Neonatal Nurs. 2007;36:511-7; quiz 518. doi:10.1111/j.1552-6909.2007.00180.x

5. Flier JS. Obesity wars: molecular progress confronts an expanding epidemic. Cell. 2004;116:337-350. doi:10.1016/S0092-8674(03)01081-X

6. Ruohonen ST, Vähätalo LH, Savontaus E. Diet-induced obesity in mice overexpressing neuropeptide y in noradrenergic neurons. Int $J$ Pept. 2012;2012:452524. doi:10.1155/2012/452524

7. Billington CJ, Levine AS. Hypothalamic neuropeptide Y regulation of feeding and energy metabolism. Curr Opin Neurobiol. 1992;2:847-851. doi:10.1016/0959-4388(92)90144-A

8. Chao PT, Yang L, Aja S, Moran TH, Bi S. Knockdown of NPY expression in the dorsomedial hypothalamus promotes development of brown adipocytes and prevents diet-induced obesity. Cell Metab. 2011;13:573-583. doi:10.1016/j.cmet.2011.02.019

9. Ishihara A, Kanatani A, Mashiko S, et al. A neuropeptide Y Y5 antagonist selectively ameliorates body weight gain and associated parameters in diet-induced obese mice. Proc Natl Acad Sci USA. 2006;103:7154-7158. doi:10.1073/pnas.0510320103

10. Acosta-Martinez M, Horton T, Levine JE. Estrogen receptors in neuropeptide Y neurons: at the crossroads of feeding and reproduction. Trends Endocrinol Metab. 2007;18:48-50. doi:10.1016/j.tem.2006.12.001

11. Heldring N, Pike A, Andersson S, et al. Estrogen receptors: how do they signal and what are their targets. Physiol Rev. 2007;87:905-931. doi:10.1152/physrev.00026.2006

12. Katzenellenbogen BS, Montano MM, Le Goff P, et al. Antiestrogens: mechanisms and actions in target cells. J Steroid Biochem Mol Biol. 1995;53:387-393. doi:10.1016/0960-0760(95)00084-D

13. Viña J, Sastre J, Pallardó FV, Gambini J, Borrás C. Role of mitochondrial oxidative stress to explain the different longevity between genders: protective effect of estrogens. Free Radic Res. 2006;40:1359-1365. doi:10.1080/10715760600952851

14. Tiidus PM. Oestrogen and sex influence on muscle damage and inflammation: evidence from animal models. Curr Opin Clin Nutr Metab Care. 2001;4:509-513. doi:10.1097/00075197-200111000-00008

15. Stubbins RE, Holcomb VB, Hong J, Núñez NP. Estrogen modulates abdominal adiposity and protects female mice from obesity and impaired glucose tolerance. Eur J Nutr. 2012;51:861-870. doi:10.1007/s00394-011-0266-4

16. Clarke BL, Khosla S. Physiology of bone loss. Radiol Clin North Am. 2010;48:483-495. doi:10.1016/j.rcl.2010.02.014

17. Pinkerton JV, Stovall DW. Reproductive aging, menopause, and health outcomes. Ann N Y Acad Sci. 2010;1204:169-178. doi:10.1111/j.1749-6632.2010.05526.x
18. Park S, Fujishita C, Komatsu T, et al. NPY antagonism reduces adiposity and attenuates age-related imbalance of adipose tissue metabolism. FASEB J. 2014;28:5337-5348. doi:10.1096/fj.14-258384

19. Martínez de Morentin PB, González-García I, Martins L, et al. Estradiol regulates brown adipose tissue thermogenesis via hypothalamic AMPK. Cell Metab. 2014;20:41-53. doi:10.1016/j.cmet.2014.03.031

20. Asarian L, Geary N. Modulation of appetite by gonadal steroid hormones. Philos Trans R Soc Lond B Biol Sci. 2006;361:1251-1263. doi:10.1098/ rstb.2006.1860

21. Klenke U, Constantin S, Wray S. Neuropeptide Y directly inhibits neuronal activity in a subpopulation of gonadotropin-releasing hormone-1 neurons via Y1 receptors. Endocrinology. 2010;151:2736-2746. doi:10.1210/ en.2009-1198

22. Aziz SA, Wakeling LA, Miwa S, Alberdi G, Hesketh JE, Ford D. Metabolic programming of a beige adipocyte phenotype by genistein. Mol Nutr Food Res. 2017;61. doi:10.1002/mnfr.201600574

23. Asano H, Kanamori Y, Higurashi S, et al. Induction of beige-like adipocytes in 3T3-L1 cells. J Vet Med Sci. 2014;76:57-64. doi:10.1292/ jvms.13-0359

24. Barros RP, Machado UF, Gustafsson JA. Estrogen receptors: new players in diabetes mellitus. Trends Mol Med. 2006;12:425-431. doi:10.1016/j. molmed.2006.07.004

25. Wohlers LM, Spangenburg EE. 17beta-estradiol supplementation attenuates ovariectomy-induced increases in ATGL signaling and reduced perilipin expression in visceral adipose tissue. J Cell Biochem. 2010;110:420-427. doi:10.1002/jcb.22553

26. Zengin A, Nguyen AD, Wong IP, et al. Neuropeptide Y mediates the short-term hypometabolic effect of estrogen deficiency in mice. Int J Obes (Lond). 2013;37:390-398. doi:10.1038/ijo.2012.71

27. Dhillon SS, Belsham DD. Estrogen inhibits NPY secretion through membrane-associated estrogen receptor (ER)- $\alpha$ in clonal, immortalized hypothalamic neurons. Int J Obes (Lond). 2011;35:198-207. doi:10.1038/ ijo.2010.124

28. Jones ME, Thorburn AW, Britt KL, et al. Aromatase-deficient (ArKO) mice have a phenotype of increased adiposity. Proc Natl Acad Sci USA. 2000;97:12735-12740. doi:10.1073/pnas.97.23.12735

29. Salpeter SR, Walsh JM, Ormiston TM, Greyber E, Buckley NS, Salpeter EE. Meta-analysis: effect of hormone-replacement therapy on components of the metabolic syndrome in postmenopausal women. Diabetes Obes Metab. 2006;8:538-554. doi:10.1111/j.1463-1326.2005.00545.x

30. Erickson JC, Ahima RS, Hollopeter G, Flier JS, Palmiter RD. Endocrine function of neuropeptide Y knockout mice. Regul Pept. 1997;70:199-202. doi:10.1016/s0167-0115(97)01007-0

31. Diaz Brinton R. Minireview: translational animal models of human menopause: challenges and emerging opportunities. Endocrinology. 2012;153:3571-3578. doi:10.1210/en.2012-1340

32. Ström JO, Theodorsson A, Ingberg E, Isaksson IM, Theodorsson E. Ovariectomy and $17 \beta$-estradiol replacement in rats and mice: a visual demonstration. J Vis Exp. 2012;e 4013. doi:10.3791/4013

33. Yasui T, Uemura H, Tezuka M, et al. Biological effects of hormone replacement therapy in relation to serum estradiol levels. Horm Res. 2001;56:3844. doi: $10.1159 / 000048088$

34. Gavaler JS. Oral hormone replacement therapy: factors that influence the estradiol concentrations achieved in a multiracial study population. J Clin Pharmacol. 2002;42:137-144. doi:10.1177/00912700222011166

35. Heine PA, Taylor JA, Iwamoto GA, Lubahn DB, Cooke PS. Increased adipose tissue in male and female estrogen receptor-alpha knockout mice. Proc Natl Acad Sci USA. 2000;97:12729-12734. doi:10.1073/ pnas.97.23.12729

36. Foryst-Ludwig A, Clemenz M, Hohmann S, et al. Metabolic actions of estrogen receptor beta (ERbeta) are mediated by a negative cross-talk with PPARgamma. PLoS Genet. 2008;4:e1000108. doi:10.1371/journal. pgen. 1000108

37. Tomicek NJ, Lancaster TS, Korzick DH. Increased estrogen receptor $\beta$ in adipose tissue is associated with increased intracellular and reduced circulating adiponectin protein levels in aged female rats. Gend Med. 2011;8:325-333. doi:10.1016/j.genm.2011.05.010 